\title{
Conjunction Challenges of Low-Thrust Geosynchronous Debris Removal Maneuvers
}

\author{
Paul V. Anderson ${ }^{\mathrm{a}, 1, *}$, Hanspeter Schaub ${ }^{\mathrm{a}, 2}$ \\ ${ }^{a}$ University of Colorado, Boulder, CO, 80309, USA
}

\begin{abstract}
The conjunction challenges of low-thrust engines for continuous thrust re-orbiting of geosynchronous (GEO) objects to super-synchronous disposal orbits are investigated, with applications to end-of-life mitigation and active debris removal (ADR) technologies. In particular, the low maneuverability of low-thrust systems renders collision avoidance a challenging task. This study investigates the number of conjunction events a low-thrust system could encounter with the current GEO debris population during a typical re-orbit to $300 \mathrm{~km}$ above the GEO ring. Sensitivities to thrust level and initial longitude and inclination are evaluated, and the impact of delaying the start time for a re-orbiting maneuver is assessed. Results demonstrate that the mean number of conjunctions increases hyperbolically as thrust level decreases, but timing the start of the maneuver appropriately can reduce the average conjunction rate when lower thrust levels are applied.
\end{abstract}

Keywords: geosynchronous orbit, orbital debris, conjunction events, continuous thrust

\section{Introduction}

The geostationary (GEO) regime is a unique commodity of the terrestrial satellite industry that is becoming increasingly contaminated with orbital debris (Johnson, 1999; Jehn et al., 2005), but is heavily populated with high-value assets (Chrystal et al., 2011). As the lack of atmospheric drag effects at the GEO altitude renders lifetimes of these debris essentially infinitely long, conjunction assessment must be performed to safeguard operational GEO satellites from potential collisions with the uncontrolled derelict field. GEO satellites must maintain a specified longitude slot, and cannot simply shift in phase to evade debris. Therefore, as the resident space object population at GEO continues to increase, the fuel cost required to remain at a particular longitude slot while performing collision avoidance with uncontrolled objects will begin to increase in tandem. Ultimately, global adherence to end-oflife mitigation guidelines must be combined with environmental remediation - active debris removal (ADR) - to curtail debris growth in this regime (Anderson and Schaub, 2014). The

\footnotetext{
*Corresponding author

Email addresses: paul.anderson@colorado.edu (Paul V. Anderson), hanspeter.schaub@colorado.edu (Hanspeter Schaub)

${ }^{1}$ Graduate Research Assistant, Department of Aerospace Engineering Sciences, 429 UCB, Boulder, CO, 80309 .

${ }^{2}$ Professor, Department of Aerospace Engineering Sciences, 429 UCB, Boulder, CO, 80309.
} 
necessity for cost-effective ADR implementation in the GEO ring is becoming more prominent, especially for larger debris (payloads, upper stages) that pose the greatest threat to operational assets.

Proposed ADR techniques for the GEO arena typically involve re-orbiting of large-scale derelicts to "graveyard" disposal orbits at perigee altitudes above the GEO ring, factoring the GEO protection zone (Flohrer, 2014) and area-to-mass ratio of the object into the minimum altitude calculation (Jehn et al., 2005; Jehn and Hernandez, 2001; NASA, 2009). A chief space-tug concept is often envisioned for performing the re-orbiting maneuver once contact with the target debris object has been established. However, as rendezvous, proximity operations, and docking with an uncontrolled - and potentially tumbling - object are challenging, several proposed methods have focused on contactless technologies such as an electrostatic tractor (Schaub and Moorer, 2012) or ion beam shepherd (Bombardelli and Pelaez, 2011) for ADR at GEO. Each of these contactless ADR technologies rely on low-thrust engines for performing the required re-orbit maneuver. With lower maneuverability, however, collision avoidance for such low-thrust re-orbit systems is challenging. Potential conjunctions must be detected multiple revolutions in advance, to give the tug guidance system enough lead time to place the tug on a sufficient evasive trajectory, especially if this craft is designed to operate autonomously with minimal ground support. Of interest is investigating how many conjunction events with the current debris population at GEO could be experienced during a typical continuous thrust re-orbiting maneuver to an IADC-compliant graveyard orbit 300 $\mathrm{km}$ above the GEO ring (Jehn et al., 2005). Specifically, quantifying the "conjunction challenge" for a particular thrust level — that is, the global average of the number of conjunctions that could be expected for a re-orbit trajectory at this thrust level, regardless of initial longitude or inclination - is a useful tool for architecture and system design for potential ADR demonstration missions at GEO.

Evaluating the global conjunction challenge for low-thrust GEO disposal maneuvers is beneficial not only for remediation concepts, but for operational end-of-life mitigation activities as well. Since lightweight, all-electric busses are becoming more prominent in the satellite industry, ${ }^{3}$ lower-thrust electric propulsion is now being harnessed both for orbit raising and station-keeping of GEO assets. Previously, GEO satellites with chemical thrusters have used a two- to three-impulse Hohmann-like transfer to re-orbit to a disposal orbit at end-of-life (Klinkrad, 2006; Raballand et al., 2005) but GEO satellites equipped with lowthrust electric engines must use continuous thrust orbit raising strategies to achieve the desired increase in semi-major axis. Since continuous thrust re-orbiting performed under $\mathrm{mN}$ levels of thrust takes from weeks to months to achieve a $300 \mathrm{~km}$ increase in semi-major axis (Schaub and Moorer, 2012), it is important to investigate the number of conjunctions that such satellites might experience during this phase of decommissioning. It is undesirable to be responsible for a debris-generating event while engaged in an act of mitigation or remediation - thus, characterizing the conjunction challenges of low-thrust re-orbit maneuvers is critical knowledge for both all-electric satellite operators and designers of low-thrust ADR systems. $^{4}$

\footnotetext{
${ }^{3}$ de Selding, P. B., "News from Satellite 2014: Boeing Electric Satellite Backlog Posed to Grow, includes Previously Undisclosed U.S. Gov't Order," Space News, 13 March 2014, Web.

${ }^{4}$ In this paper, GEO satellites using continuous thrust during end-of-life operations, and ADR space-tug
} 


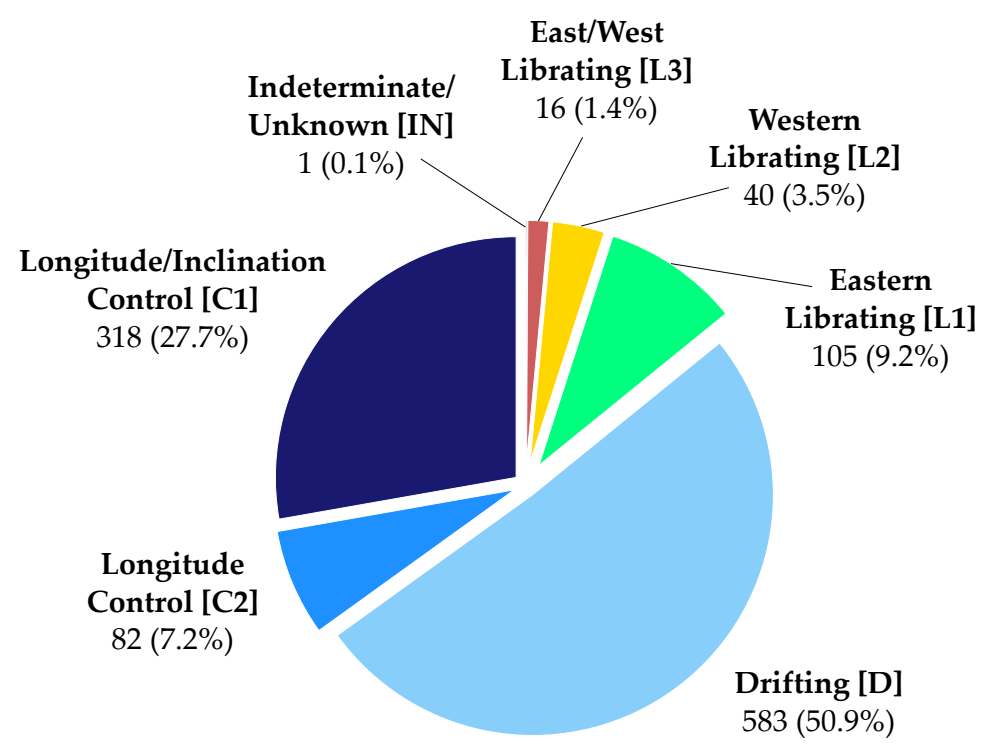

Figure 1: GEO orbit classifications for 02/28/2014 reference TLE set.

Table 1: Orbit classifications for geosynchronous objects used in GEO conjunction study.

\begin{tabular}{c|c|l} 
Class & Type & \multicolumn{1}{|c}{ Description } \\
\hline C1 & Controlled & Longitude/inclination control (E-W/N-S control) \\
C2 & Controlled & Longitude control only (E-W control only) \\
D & Drifting & Circulating above/below/through GEO altitude \\
L1 & Librating & Libration about Eastern stable point at $75^{\circ} \mathrm{E}$ \\
L2 & Librating & Libration about Western stable point at $105^{\circ} \mathrm{W}$ \\
L3 & Librating & Libration about Eastern and Western stable points \\
IN & Indeterminate & Unknown status (e.g., recent TLE not available)
\end{tabular}

\section{Current Resident Space Object (RSO) Population at GEO}

The RSO population in the GEO regime is classified with a taxonomy used by the European Space Agency's DISCOS database (Database and Information System Characterising Objects in Space) (Flohrer, 2014). For GEO objects, seven orbit categories are selected to classify the type of orbits traversed by these RSOs - two controlled classes and five uncontrolled classes. Note that only uncontrolled objects are assumed to contribute to local debris congestion in this study. GEO RSOs are selected according to the orbit element bounds used in the European Space Agency's Classification of Geosynchronous Objects reports (Flohrer, 2014): eccentricity less than $0.2(e<0.2)$, inclination less than $70^{\circ}\left(i<70^{\circ}\right)$, and mean motion between $0.9-1.1$ revs per sidereal day $(0.9<n<1.1)$, corresponding to the semi-major axis range $-2596 \mathrm{~km}<a<3068 \mathrm{~km}$ with respect to GEO.

Orbital data is obtained from the publicly-available two-line element (TLE) sets provided

concepts that use continuous thrust to re-orbit derelict objects to disposal orbits, are collectively termed re-orbit systems for generality. 
by U.S. Strategic Command (USSTRATCOM). ${ }^{5}$ For this study, a reference TLE set obtained on $02 / 28 / 2014$ is employed; the class distribution for the 1145 objects extracted from this set is shown in Figure 1. TLE data are provided in the form of doubly-averaged Keplerian elements with mean motion instead of semi-major axis (Klinkrad, 2006), transformed into Cartesian states in the true equator, mean equinox (TEME) frame (Vallado, 2007) with SGP4 theory (Hoots and Roehrich, 1980). ${ }^{6}$ Note that because of the limited accuracy of TLE data sets, these data are not intended for studies that require highly-precise orbit prediction capabilities. Furthermore, as only objects larger than approximately 0.8-1.0 meter in effective diameter are actively tracked at the GEO altitude (Flohrer, 2014), only objects at least of this size are considered here. Since this analysis only includes the trackable, catalogued, and unclassified GEO population with recent TLE sets, the findings of this study serve to illustrate a lower bound of the actual potential for conjunctions during GEO re-orbit.

\section{Analytic Results for Continuous Thrust Trajectories}

In the framework of two-body mechanics, analytic expressions that describe the semimajor axis and longitude profiles for a continuous thrust re-orbit trajectory - as a function of thrust acceleration and elapsed time since the start of the re-orbit maneuver - are now derived. Following Prussing and Conway (2013), the temporal derivative of the specific two-body orbit energy is

$$
\epsilon=-\frac{\mu_{\oplus}}{2 a} \quad \Longrightarrow \quad \dot{\epsilon}=\frac{\mu_{\oplus}}{2 a^{2}} \dot{a}
$$

where $\dot{a}$ denotes the time rate of change of the semi-major axis. From elementary physics, the rate of change of specific energy due to a thrust vector $\boldsymbol{\Gamma}$ is $\dot{\epsilon}=\boldsymbol{\Gamma} \cdot \boldsymbol{v}$, where $\boldsymbol{v}$ is the inertial velocity vector. Assuming that the thrust acceleration is directed along the instantaneous velocity direction, we have $\dot{\epsilon}=\Gamma v$, such that

$$
\frac{\mu_{\oplus}}{2 a^{2}} \dot{a}=\Gamma v \quad \Longrightarrow \quad \dot{a}=\frac{2 a^{2} \Gamma}{\mu_{\oplus}} v \approx \frac{2 a^{3 / 2} \Gamma}{\sqrt{\mu_{\oplus}}}
$$

where it is assumed that the osculating orbit remains approximately circular during the continuous-thrust re-orbit, such that $v=\sqrt{\mu_{\oplus} / a}$ is applicable. Separating variables and assuming constant $\Gamma$, we have:

$$
\int a^{-3 / 2} \mathrm{~d} a=\frac{2 \Gamma}{\sqrt{\mu_{\oplus}}} \int \mathrm{d} t
$$

Performing the integration and enforcing the initial condition $a(0)=a_{0}$ :

$$
-\frac{1}{\sqrt{a}}=\frac{\Gamma}{\sqrt{\mu_{\oplus}}} t-\frac{1}{\sqrt{a_{0}}} \Longrightarrow a(t)=\frac{a_{0} \mu_{\oplus}}{\left(\sqrt{\mu_{\oplus}}-\Gamma \sqrt{a_{0}} t\right)^{2}}
$$

\footnotetext{
${ }^{5}$ Publicly-available TLE data sets are available for bulk download from https://www . space-track.org/ ${ }^{6} \mathrm{C}$ implementation of SGP-4/SDP-4 is available at http://www.sat.dundee.ac.uk/ psc/sgp4. html (Vallado et al., 2006)
} 
Thus, the time $t_{f}$ required to transfer between circular orbits of radii $a_{0}$ and $a_{f}>a_{0}$ is given by (Prussing and Conway, 2013):

$$
t_{f}=\frac{\sqrt{\mu_{\oplus}}}{\Gamma}\left(\frac{1}{\sqrt{a_{0}}}-\frac{1}{\sqrt{a_{f}}}\right)=\frac{v_{0}-v_{f}}{\Gamma}
$$

As a result, $v_{f}=v_{0}-\Gamma t_{f}$, that is, the re-orbit system slows down linearly in time as local circular orbit speed is maintained (Prussing and Conway, 2013). Figure 2 illustrates Equation (5) as a function of thrust acceleration for three super-synchronous semi-major axes. The hyperbolic form of Equation (5) indicates that as the thrust acceleration increases, the length of the re-orbit maneuver decreases rapidly. Furthermore, as the target semi-major axis $a_{f}$ increases, local orbit speed $v_{f}$ at $a_{f}$ decreases, such that the length of the re-orbit maneuver increases. As will be shown, the duration of the re-orbit maneuver is strongly correlated to the number of conjunction events a trajectory at a given thrust acceleration experiences.

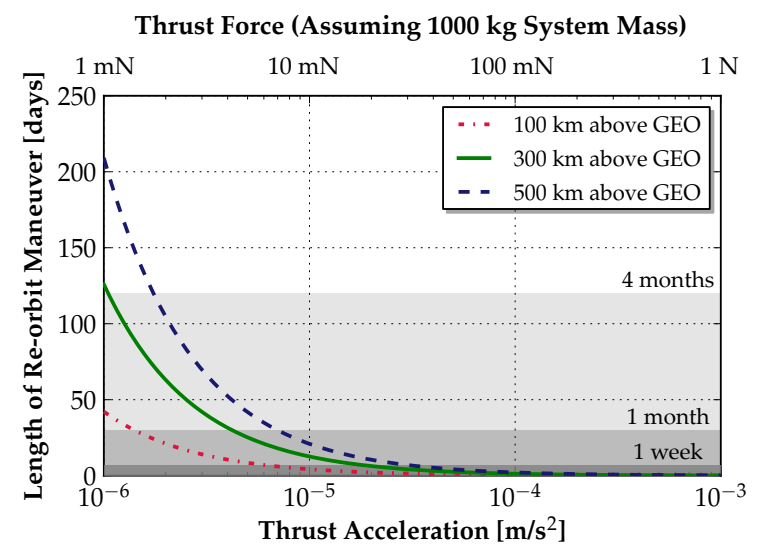

(a) Analytic re-orbit duration (linear $y$-axis).

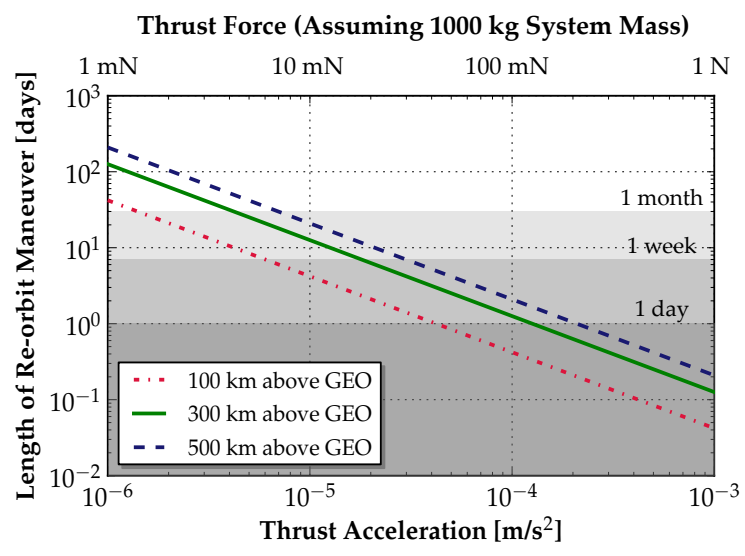

(b) Analytic re-orbit duration (logarithmic $y$ axis).

Figure 2: Duration of re-orbit to various semi-major axes above GEO as a function of thrust level.

For a near-circular orbit, the rate of change of longitude $\dot{\lambda}$ is given by the mean motion difference

$$
\dot{\lambda}=n-n_{\mathrm{GEO}}=\sqrt{\frac{\mu_{\oplus}}{a^{3}}}-\sqrt{\frac{\mu_{\oplus}}{a_{\mathrm{GEO}}^{3}}}
$$

Substituting Equation (4), the longitude rate becomes

$$
\dot{\lambda}(t)=\frac{\left(\sqrt{\mu_{\oplus}}-\Gamma \sqrt{a_{0}} t\right)^{3}}{a_{0}^{3 / 2} \mu_{\oplus}}-\sqrt{\frac{\mu_{\oplus}}{a_{\mathrm{GEO}}^{3}}}
$$

Integrating Equation (7) and enforcing the initial condition $\lambda(0)=\lambda_{0}$ :

$$
\lambda(t)=\frac{\mu_{\oplus}^{2}-\left(\sqrt{\mu_{\oplus}}-\Gamma \sqrt{a_{0}} t\right)^{4}}{4 \Gamma a_{0}^{2} \mu_{\oplus}}-\sqrt{\frac{\mu_{\oplus}}{a_{\mathrm{GEO}}^{3}}} t+\lambda_{0}
$$


Given that the re-orbiting system begins at longitude $\lambda_{0}$, Equation (8) provides an analytic prediction for the resulting longitude profile of the system as a function of elapsed time $t$ since maneuver start, initial semi-major axis $a_{0}$, and thrust acceleration $\Gamma$. Since the semi-major axis increases above that of GEO during the re-orbit maneuver, as described by Equation (4), the mean motion of the system slows with respect to the rotation rate of Earth, resulting in westward longitudinal drift. To evaluate the total amount of longitudinal drift $\Delta \lambda \equiv \lambda\left(t_{f}\right)-\lambda_{0}$ that occurs during a re-orbit as a function of thrust acceleration, the maneuver duration $t_{f}$ in Equation (5) is substituted into Equation (8), giving

$$
\Delta \lambda=\frac{\mu}{4 \Gamma a_{0}^{2}}\left[1-\left(\frac{a_{0}}{a_{f}}\right)^{2}\right]-\sqrt{\frac{\mu_{\oplus}}{a_{\mathrm{GEO}}^{3}}}\left(\frac{v_{0}-v_{f}}{\Gamma}\right)
$$

Figure 3 illustrates Equation (9) as a function of thrust level for three super-synchronous semi-major axes. The hyperbolic form of Equation (9) indicates that as the thrust acceleration increases, the total longitudinal drift accumulated over the duration of a re-orbit decreases rapidly. Further, since larger $a_{f}$ require longer maneuver durations $t_{f}$ by Equation (5), total longitudinal drift increases with $a_{f}$ as a result. Figure 3 provides an interesting compliment to continuous-thrust trajectory representations in the inertial frame, which exhibit a "spiral-like" pattern in inertial space. For simulated re-orbits to $300 \mathrm{~km}$ above GEO, Figure 3 shows that the re-orbit system does not complete an entire revolution in the Earthfixed frame over the range of thrust accelerations considered. This has implications for ground station coverage of these re-orbit maneuvers, and highlights that systems beginning in less-congested slots could still pass through heavily-congested longitudes over the course of the transfer.

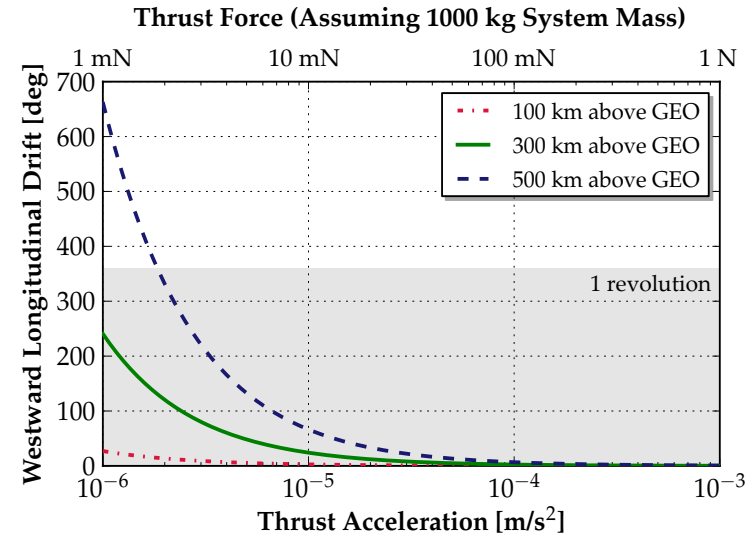

(a) Analytic longitude drift (linear $y$-axis).

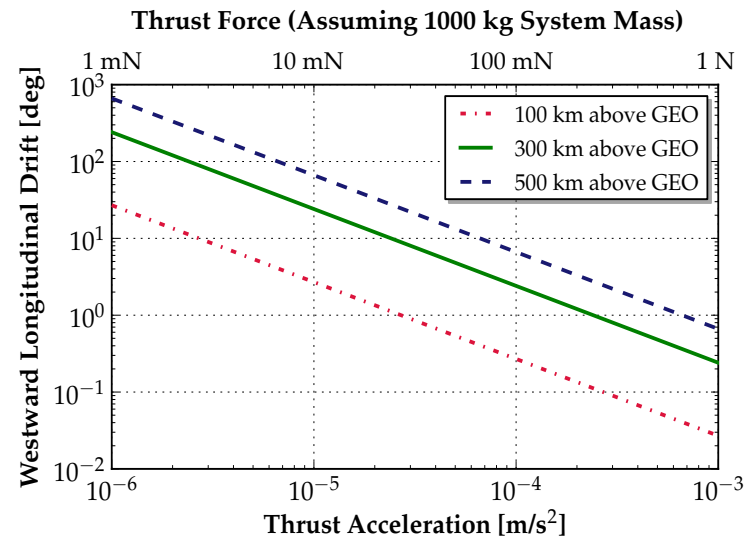

(b) Analytic longitude drift (logarithmic $y$-axis).

Figure 3: Total drift in longitude for various re-orbit maneuvers as a function of thrust level.

\section{Evaluating Conjunctions for GEO Re-orbit Maneuvers}

To begin developing insight into the conjunction challenges for continuous thrust re-orbit systems at GEO, this study considers the number of conjunction events that a re-orbit system 
could encounter with the current GEO debris population during a typical re-orbit maneuver to $300 \mathrm{~km}$ above the GEO ring. Specifically, the research questions investigated in this GEO conjunctions study are as follows:

- How many conjunctions within various distances can be expected, on average, for a specified thrust level, ranging from $10^{-6}$ to $10^{-3} \mathrm{~m} / \mathrm{s}^{2}$ (i.e., $1 \mathrm{mN}$ to $1 \mathrm{~N}$ thrust for a $1000 \mathrm{~kg}$ system)?

- Is the number of conjunctions dependent on initial longitude and inclination, and if so, is this dependency sensitive to the thrust level or the time at which the re-orbiting maneuver begins?

- When are these conjunctions most likely to occur during the re-orbit burn, regardless of initial longitude or inclination? Does the likelihood of a conjunction increase over a range of semi-major axes, and if so, which classes of debris objects are responsible for these conjunctions?

- What is the distribution of these conjunction events in the Hill frame for a given thrust level? What are the relative velocities associated with the conjunction events for a given thrust level?

\subsection{Propagator and Implementation}

A special perturbations propagator implemented in ANSI-C and parallelized with OpenCL is used to propagate the GEO debris population forward in time and detect conjunction events during a low-thrust re-orbit maneuver. ${ }^{7}$ The two-body equations of motion are numerically integrated under $4 \times 4$ EGM-96 gravitation, luni-solar perturbations, and solar radiation pressure $(\mathrm{SRP})$, modeled with the cannonball assumption described in Vallado (2007), and attenuated with the occultation algorithm in Montenbruck and Gill (2000). The equations of motion for the uncontrolled GEO debris objects are

$$
\ddot{\boldsymbol{r}}=-\frac{\mu_{\oplus}}{r^{3}} \boldsymbol{r}+\boldsymbol{a}_{\oplus}+\boldsymbol{a}_{\overparen{ }}+\boldsymbol{a}_{\odot}+\boldsymbol{a}_{\mathrm{SRP}}
$$

where the first term denotes two-body acceleration, $\boldsymbol{a}_{\oplus}$ is the acceleration due to the nonsphericity of Earth, $\boldsymbol{a}_{\varangle}$ and $\boldsymbol{a}_{\odot}$ are the third-body perturbations from the Moon and Sun, respectively, and $\boldsymbol{a}_{\mathrm{SRP}}$ is the SRP acceleration. SRP is modeled using the inverse-square diffusion formulation of the solar luminosity $L_{\odot} \approx 3.839 \times 10^{26} \mathrm{~J} / \mathrm{s}$, with coefficient of reflectivity $c_{r} \equiv 1.5$ and GEO-representative area-to-mass ratio $A_{\odot} / m=0.04 \mathrm{~m}^{2} / \mathrm{kg}^{8}{ }^{8}$ This GEO force model is in agreement with the results of Hansen and Sorge (2013), who rank the importance of incorporating various environmental perturbations in GEO-specific forcing models for debris analysis over time scales ranging from 1 week to 10 years.

\footnotetext{
${ }^{7}$ The OpenCL 1.2 Specification is available at: http://www.khronos.org/registry/cl/.

${ }^{8}$ Schaub and Jasper (2011) indicate that this ratio is representative for operational and derelict satellites at GEO - this value is thus used in the SRP computation for all objects considered in this study. Furthermore, a changing area-to-mass ratio for the re-orbit system is neglected, since mass loss during re-orbit is not significant even for the lowest thrust level considered.
} 
The equations of motion for the re-orbit system are equivalent to those for the uncontrolled objects, with the addition of a continuous thrust acceleration vector $\boldsymbol{a}_{t}$, which is oriented in the in-track direction of the local orbit frame: ${ }^{9}$

$$
\ddot{\boldsymbol{r}}=-\frac{\mu_{\oplus}}{r^{3}} \boldsymbol{r}+\boldsymbol{a}_{\oplus}+\boldsymbol{a}_{\overparen{ }}+\boldsymbol{a}_{\odot}+\boldsymbol{a}_{\mathrm{SRP}}+\boldsymbol{a}_{t}
$$

Both propagators harness an eighth-order, predictor-corrector Gauss-Jackson method (Berry and Healy, 2004) initialized with the Prince-Dormand 8(7) algorithm for integration of the equations of motion in Equations (10)-(11). The debris population is propagated from 03/01/2014 (00:00:00 Zulu) for 5 months in 5 minute time steps, and each continuous thrust re-orbit trajectory is propagated forward from the same epoch, until $300 \mathrm{~km}$ above GEO in osculating semi-major axis is achieved. Conjunctions are then detected in post-processing by checking for debris objects that come within a specified distance threshold of the re-orbit system at any time step over the duration of the maneuver. Logic is applied to ensure that multiple conjunctions from the same object detected over subsequent steps are only counted once.

\subsection{Risk Functions for Relative Position and Velocity}

To quantify how threatening simulated conjunction events are for a re-orbit system, each conjunction event is assigned a level of combined risk based upon the relative state vector of the responsible debris object at the time of conjunction. Position and velocity risk factor functions are employed to individually weight (a) how close the object comes to the re-orbit system, and (b) how fast the object is traveling relative to the re-orbit system. Specifically, the relative position and velocity risk factor functions selected for this study are (Anderson and Schaub, 2016)

$$
\begin{aligned}
& R_{r}(r)=\left(\frac{\tilde{r}-r}{\tilde{r}}\right)^{2}, \quad 0 \leqslant r \leqslant \tilde{r} \\
& R_{v}(v)=1-e^{-3 v / \bar{v}}, \quad v \geqslant 0
\end{aligned}
$$

where $\tilde{r}$ denotes the conjunction distance threshold, and $r$ and $v$ denote the magnitude of the relative position and velocity vectors, respectively. ${ }^{10}$ The position risk factor function in Equation (12) is of quadratic form, rising smoothly from $R_{r}(\tilde{r})=0$ at the conjunction threshold $\tilde{r}$ to $R_{r}(0)=1$ at the position of the re-orbit system. The velocity risk function in Equation (13) is of exponential form, selected to rapidly saturate to $R_{v}(v) \rightarrow 1$ when the relative velocity surpasses a defined threshold, beyond which collision velocities are considered "catastrophic." In this manner, all relative velocities above this threshold are weighted nearly equally, since a collision with a debris object traveling with a catastrophic relative velocity would have serious, mission-ending consequences for a re-orbit system (especially while

\footnotetext{
${ }^{9}$ A simplified, constant acceleration model is applied for generality of results, i.e., such that system masses and propulsive system parameters - which drive mass flow rate - need not be specified in this formulation.

${ }^{10}$ Given that the inertial frame positions and velocities for both the re-orbit system and a conjuncting debris object are known at the time of conjunction, the relative position and velocity of the debris object can be described in the local Hill frame using the relative motion algorithm in Schaub and Junkins (2009).
} 
performing mitigation or ADR), regardless of where this velocity lies above the catastrophic threshold.

The relative velocity is scaled by the time-constant-like parameter $\bar{v}$ in Equation (13), such that if $v=\bar{v}$, the risk function $R_{v}(\bar{v})=1-e^{-3} \approx 0.950$ and begins saturating. For this analysis, the parameter $\bar{v}$ is derived from the NASA Standard Breakup Model (Johnson et al., 2001), which was developed for NASA's long-term debris environment software EVOLVE 4.0, and has been validated against catalogued debris clouds and ground-based experimental results for particles larger than $1 \mathrm{~mm}$ (Klinkrad, 2006). In particular, $\bar{v}$ is selected as the relative speed threshold above which on-orbit collisions become catastrophic (i.e., complete disintegration of both objects), assuming equal masses $m_{t}$ and $m_{p}$ for the target and impactor objects in the collision event, respectively (Johnson et al., 2001):

$$
\tilde{E}_{p}^{*}=\frac{1}{2}\left(\frac{m_{p}}{m_{t}}\right) \bar{v}^{2} \rightarrow \bar{v}=\sqrt{\frac{2 \tilde{E}_{p}^{*} m_{t}}{m_{p}}} \approx 0.283 \mathrm{~km} / \mathrm{s}
$$

where $\tilde{E}_{p}^{*} \equiv 40 \mathrm{~kJ} / \mathrm{kg}$ is the specific energy threshold for a catastrophic collision (Klinkrad, 2006). Hanada et al. (2005) compare the predictions of the NASA Standard Breakup Model against low-velocity impact experiments performed at a velocity range less than $0.3 \mathrm{~km} / \mathrm{s}$, and conclude that the hypervelocity collision model in the NASA Standard Breakup Model can be applied to lower-velocity collisions, with minor modifications that do not affect Equation (14). An alternate catastrophic collision threshold introduced by McKnight (1991) is $10 \bar{v}^{2} m_{p} \geqslant m_{t}$ on a qualitative basis - applying $m_{p}=m_{t}$ as before, $\bar{v} \approx 0.316 \mathrm{~km} / \mathrm{s}$, close to the catastrophic collision threshold of $0.283 \mathrm{~km} / \mathrm{s}$ in Equation (14) per the NASA Standard Breakup Model.

After the relative position and velocity for each detected conjunction event are computed, the risk factor functions defined via Equations (12)-(13) are evaluated, such that the combined risk factor given by the product $R_{r}(r) R_{v}(v)$ is evaluated. This combined risk factor for the conjunction event is on the interval $[0,1]$ and provides a metric for gauging how "threatening" the conjunction event is for the re-orbit system. Note that both close proximity and sufficient relative speed must be present for a conjunction event to be considered high-risk under this metric - both higher-speed events near the conjunction distance threshold and lower-speed events in close proximity to the re-orbit system are de-weighted in the combined risk factor.

\section{Results of Conjunctions Study}

\subsection{Global Conjunction Challenge}

To evaluate the global conjunction challenge for a particular thrust level, a two-dimensional sweep over initial longitude on $\left[0^{\circ}, 360^{\circ}\right)$ and inclination on $\left[0^{\circ}, 15^{\circ}\right]$ is performed in $5^{\circ}$ and $1^{\circ}$ increments, respectively. The re-orbit system is initialized with $a=a_{\mathrm{GEO}}, e=0.001$, $\omega=M_{0}=0^{\circ}$, and a right ascension of ascending node selected to enforce the initial longitude at the start epoch of 03/01/2014 (00:00:00 Zulu). Figure 4 illustrates the mean number of conjunctions per trajectory at various thrust levels and distance thresholds, globally averaged over the 1152 trajectories propagated for each of the seven thrust accelerations surveyed, ranging from $10^{-6}$ to $10^{-3} \mathrm{~m} / \mathrm{s}^{2}$ (i.e., $1 \mathrm{mN}$ to $1 \mathrm{~N}$ of thrust for a $1000 \mathrm{~kg}$ system). For all distances considered, the mean number of conjunctions per trajectory rises hyperbolically as the thrust level decreases, a consequence of the result that re-orbit duration to $300 \mathrm{~km}$ 
above GEO increases hyperbolically as the thrust level decreases (cf. Figure 2). Therefore, the lower the thrust level considered for the re-orbit system, the more "challenging" the maneuver becomes from the perspective of conjunction potential - the burn time is lengthened, such that more conjunctions with the large-scale derelict population are possible over the duration of the maneuver. Note that for the $500 \mathrm{mN}$ and $1 \mathrm{~N}$ levels, no conjunctions beneath $25 \mathrm{~km}$ are experienced across all 1152 trajectories surveyed at these thrust levels, emphasizing that the mean number of conjunctions anticipated at a given thrust level and miss distance becomes nearly zero as the thrust level increases.

In addition to quantifying the mean number of conjunctions per trajectory, it is also of interest to evaluate the standard deviation $\sigma$ for the conjunction distributions generated at each thrust level and distance threshold. Figure 4(a) illustrates $1 \sigma$ swaths for each thrust level and distance, highlighting that the $1 \sigma$ spread of these conjunction distributions decreases as the thrust increases at each distance threshold. Therefore, the number of conjunctions anticipated for a given trajectory at the $1 \mathrm{mN}$ level, for example, becomes more uncertain in that this trajectory could experience fewer - or conversely, many more - conjunctions at a distance threshold of $50 \mathrm{~km}$ than the global average of approximately 2.7 conjunctions per trajectory at this distance. This result is further emphasized in the next section.

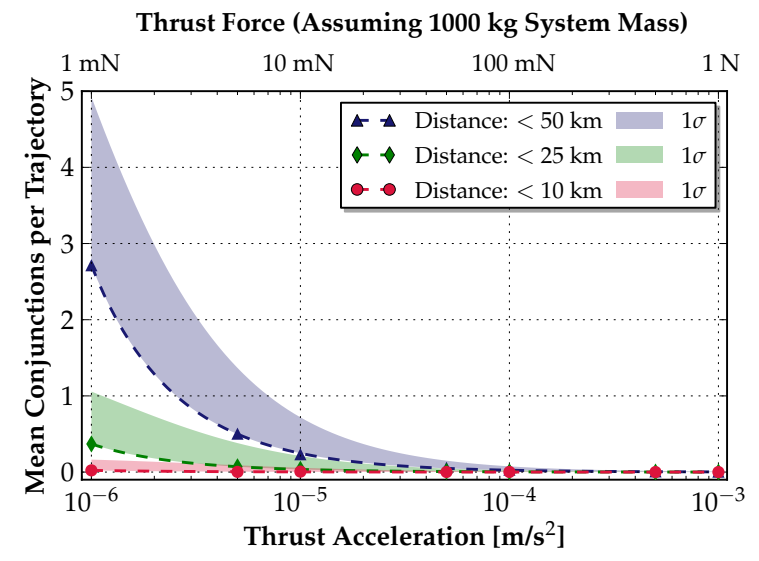

(a) Mean number of conjunctions with $1 \sigma$ swaths.

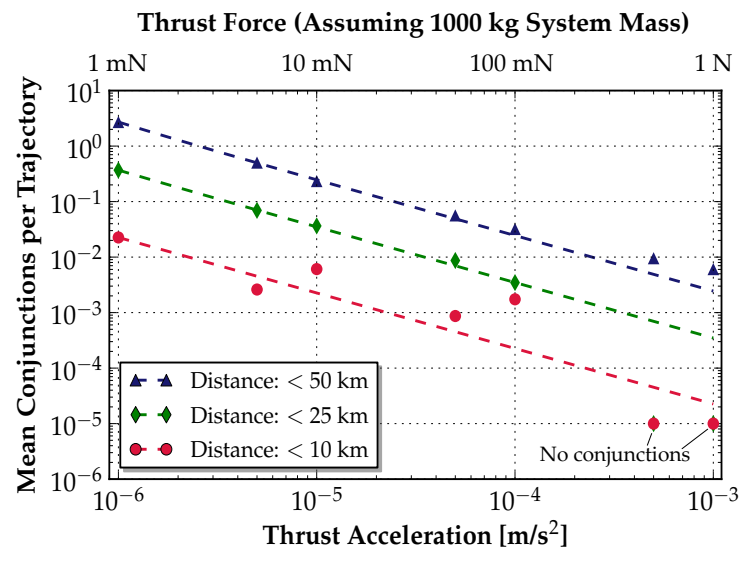

(b) Mean number of conjunctions (logarithmic $y$ axis).

Figure 4: Mean number of conjunction events per trajectory as a function of thrust level and distance, shown with hyperbolic best-fit lines on linear and logarithmic axes.

\subsection{Dependence on Initial Longitude and Inclination}

Next, it is of interest to evaluate whether the number of conjunctions experienced for a particular trajectory at a particular thrust acceleration is sensitive to the initial longitude and inclination of the re-orbit system at the beginning of the maneuver. Figure 5 illustrates the number of conjunctions at a distance threshold of $50 \mathrm{~km}$ experienced for each of the 1152 trajectories surveyed at $1 \mathrm{mN}, 5 \mathrm{mN}$, and $10 \mathrm{mN}$ thrust accelerations. Qualitative trends in the number of conjunction events experienced at each thrust acceleration are not observed, indicating that there is no deterministic dependence of the number of anticipated conjunctions on the initial longitude and inclination of the re-orbit system. As the thrust 


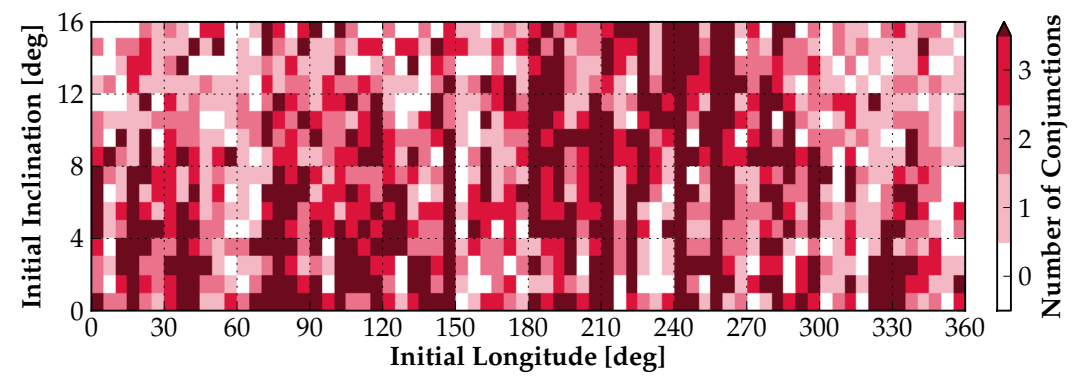

(a) Number of conjunctions at $50 \mathrm{~km}$ (thrust level: $1.0 \times 10^{-6} \mathrm{~m} / \mathrm{s}^{2}$ ).

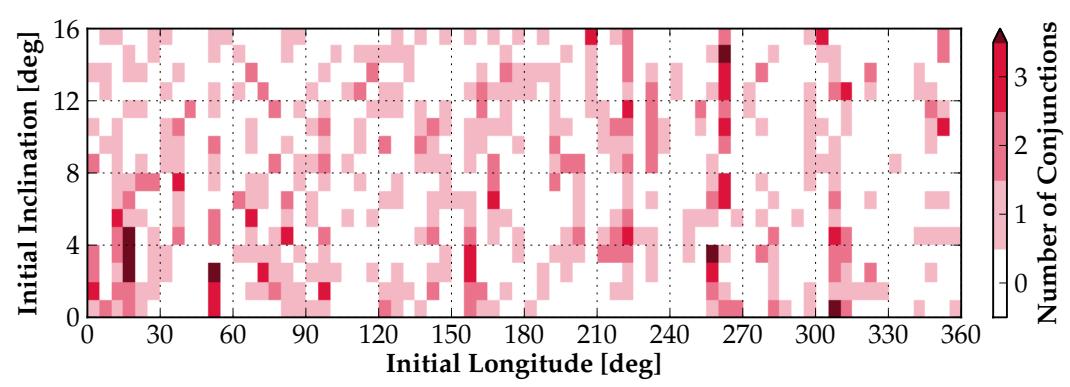

(b) Number of conjunctions at $50 \mathrm{~km}$ (thrust level: $5.0 \times 10^{-6} \mathrm{~m} / \mathrm{s}^{2}$ ).

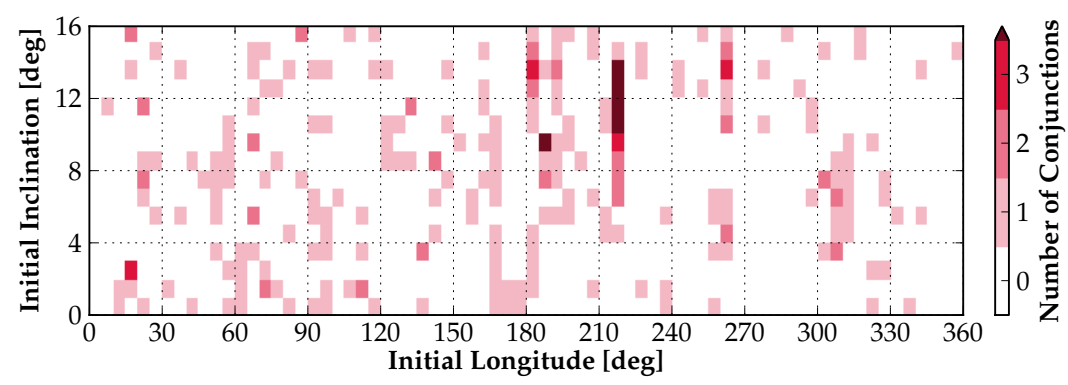

(c) Number of conjunctions at $50 \mathrm{~km}$ (thrust level: $1.0 \times 10^{-5} \mathrm{~m} / \mathrm{s}^{2}$ ).

Figure 5: Number of conjunctions at $50 \mathrm{~km}$ as a function of initial longitude and inclination.

acceleration increases, "holes" in the initial condition space begin appearing in greater frequency, corroborating the result of Figure 4 that the number of conjunctions experienced globally for a given thrust acceleration decreases hyperbolically as this thrust increases. Although the global average in Figure 4 implies that the number of conjunctions is sparse at all distances considered for thrust levels larger than $\sim 5 \mathrm{mN}$, Figure 5 (c) illustrates that particular regions of the initial condition space could still be subject to upwards of three conjunction events at $50 \mathrm{~km}$ over the course of the re-orbit. Thus, it is critical to emphasize that Figure 4 provides a first-order - not exhaustive - design tool for forecasting the number of conjunctions that could be experienced at a particular thrust level.

\subsection{Conjunctions in SMA and Longitude Space}

In addition to investigating the dependence of the number of conjunctions on the initial longitude and inclination of the re-orbit system, it is important to quantify where conjunction events are most likely to occur over the duration of the maneuver. Instead of studying 
the frequency of conjunctions as a function of elapsed time since maneuver start, it is more consistent to consider the distribution of conjunctions in semi-major axis (SMA) and longitude space. A conjunction probability for various regions in SMA and longitude space is evaluated by binning conjunction events into $25 \mathrm{~km}$ and $10^{\circ}$ bins for the SMA and longitude at which these conjunctions occur, and then by dividing the accumulated frequency in each bin by the total number of conjunctions detected at a distance threshold of $50 \mathrm{~km}$ across all thrust levels considered. Figure 6 illustrates this conjunction probability for various regions within SMA and longitude space. ${ }^{11}$ The contributions of the librating derelict population are evident around the two gravitational wells at $75^{\circ} \mathrm{E}$ and $105^{\circ} \mathrm{W}$, with GEO-relative semimajor axes from 0-50 km above the GEO ring. As the SMA increases, neighborhoods with higher conjunction probabilities are experienced, indicative of contributions by the westward drifting population at these higher altitudes.

The conjunction probability map shown in Figure 6 is an important tool in that it can be combined a priori with the analytic SMA and longitude predictions in Equations (4) and (8) to forecast when a re-orbit system will be entering a region in the phase space with a higher probability of conjunction relative to surrounding regions in this phase space. Figure 6 further shows three re-orbit trajectories beginning at a longitude of $255^{\circ}$, with $1 \mathrm{mN}, 5 \mathrm{mN}$, and $10 \mathrm{mN}$ of thrust acceleration, respectively. Given that the thrust acceleration and initial location within this phase space are known, the resulting re-orbit trajectory can be predicted using Equations (4) and (8), such that increased ground tracking can be requested prior to the re-orbit system entering a region with a higher conjunction likelihood (or, in the case of an autonomous ADR system, environmental sensing can be applied or augmented).

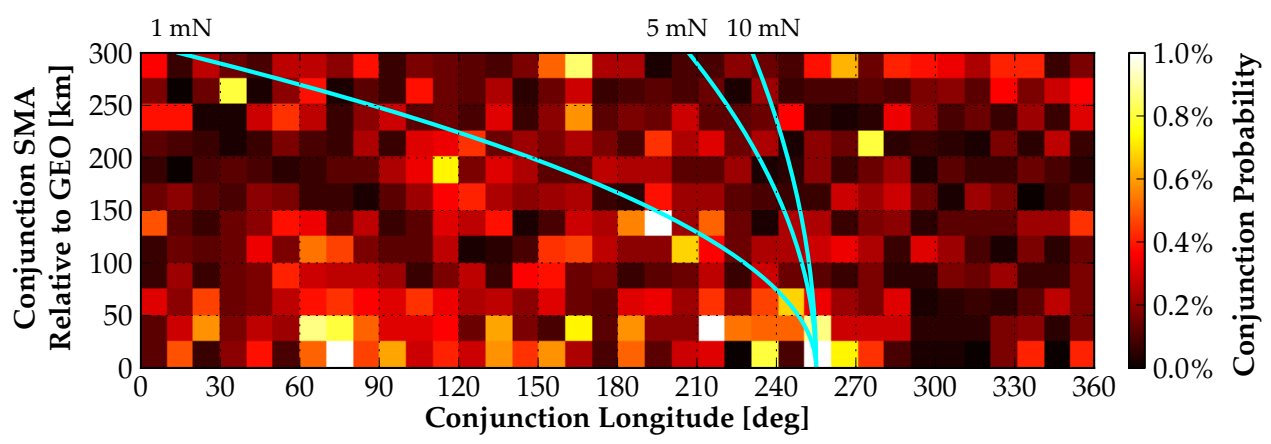

Figure 6: Density map showing regions in SMA/longitude space that experience high frequencies of conjunction events at $50 \mathrm{~km}$, illustrated with three re-orbit trajectories at various thrust levels from $255^{\circ}$ initial longitude, predicted by Equations (4) and (8).

If the conjunction probabilities shown in Figure 6 are binned by semi-major axis or longitude of conjunction only, the conditional, cumulative probability distributions for these two phase space variables - shown in Figure 7 - can be constructed by accumulating the absolute probabilities in Figure 6 across $[0,300] \mathrm{km}$ and $\left[0^{\circ}, 360^{\circ}\right)$ domains for GEO-relative SMA and longitude, respectively. In particular, Figure 7(a) highlights that the conditional cumulative probability of conjunction in SMA achieves $50 \%$ at approximately $120 \mathrm{~km}$ in SMA above the

\footnotetext{
11 "Longitude" refers here to the longitude at which each conjunction occurs, not to the initial longitude for the re-orbit system, as illustrated in Figure 5.
} 
GEO ring across all thrust levels and distance thresholds considered. Therefore, the conditional distribution of conjunctions in SMA space is not uniform; rather, it exhibits moderate bias towards the beginning of the re-orbit maneuver, a consequence of the higher densities of librating objects in closer proximity to the GEO altitude. If equipped with a variable-thrust propulsion system, the re-orbit system could reduce or potentially eliminate the number of anticipated conjunction events by increasing the thrust level during the initial stages of the re-orbit, such that the system achieves a $120 \mathrm{~km}$ SMA increase as rapidly as possible. Then, the thrust acceleration could be decreased to conserve propellant until the desired altitude is achieved. Interestingly, the conditional cumulative probability of conjunction in longitude in Figure 7(b) does not show strong contributions from the two gravitational wells, since these longitudinal regions are primarily significant for conjunction probabilities at lower altitudes in closer proximity to GEO (cf. Figure 6).

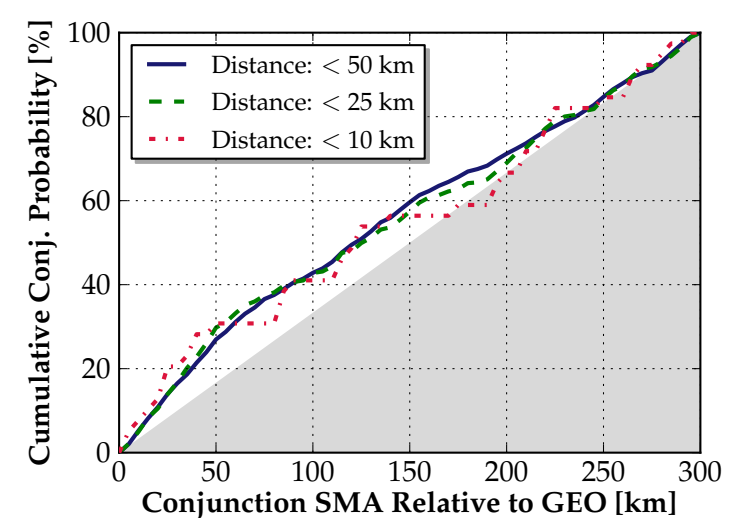

(a) Conditional cumulative probability distribution in GEO-relative SMA.

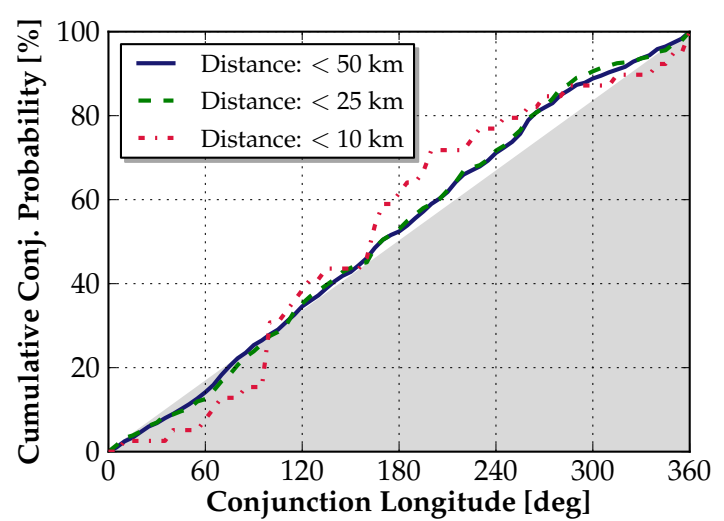

(b) Conditional cumulative probability distribution in longitude.

Figure 7: Conditional cumulative conjunction probability distributions as a function of GEO-relative semimajor axis and longitude during re-orbit (shown with uniform cumulative distribution functions for comparison).

\subsection{Relative Velocity and Risk Considerations}

In addition to the number of conjunctions observed at a given thrust level, the dependence of this number of conjunctions on the initial conditions of the re-orbit system, and where these conjunctions occur in SMA/longitude space, it is important to consider the relative velocities and combined risk factors for these conjunction events. For the $1 \mathrm{mN}$ thrust level, Figure 8 illustrates three portraits of the conjunctions observed at a distance threshold of $50 \mathrm{~km}$ across the 1152 trajectories surveyed at this thrust level: the number of conjunction events in Figure 8(a), the maximum relative velocity of the conjunctions detected for each trajectory in Figure 8(b), and the maximum combined risk of the conjunctions detected for each trajectory in Figure $8(\mathrm{c})$. Figure 8 dictates that although a particular re-orbit trajectory could experience multiple conjunction events beneath $50 \mathrm{~km}$ over the duration of the re-orbit, the worst-case relative speed for these conjunctions - or the worst-case combined risk thereof - could be benign, indicating that evasive action by the re-orbit system may not be necessary. Conversely, Figure 8 shows cases in which although only 1-2 conjunctions are 
experienced over the duration of the maneuver, these conjunctions carry a worst-case risk that may be significant enough to warrant evasive action (conjunctions are threatening via the combination of proximity and speed).

To complement the global conjunction challenge illustrated in Figure 4, Figure 9 shows the mean number of conjunctions per trajectory at various thrust levels and risk thresholds, globally averaged over the 1152 trajectories propagated for each thrust level. For each risk threshold, the mean number of conjunctions per trajectory rises hyperbolically as the thrust level decreases. As the risk threshold increases, however, the mean number of conjunctions per trajectory for a given thrust level decreases in a manner that is analogous to decreasing the conjunction distance threshold (cf. Figure 4). Thus, at the $1 \mathrm{mN}$ level, although the global conjunction challenge is approximately 2.7 conjunctions per trajectory on average at a distance threshold of $50 \mathrm{~km}$, less than 0.5 conjunctions per trajectory on average are contributed by conjunctions with a combined risk greater than 0.2 . This global average is analogous to that at a conjunction distance threshold of $25 \mathrm{~km}$ for the $1 \mathrm{mN}$ thrust level in Figure 4.

To highlight the $1 \sigma$ spread of the conjunction distributions generated for each thrust level and risk threshold, Figure 9 (a) illustrates the $1 \sigma$ swaths in addition to the mean number of conjunctions per trajectory determined at each thrust level and risk threshold. Analogous to Figure 4(a), the standard deviation decreases as the thrust level increases at a given risk threshold, and furthermore decreases as the risk threshold increases at a given thrust level. Thus, the mean number of conjunctions with a combined risk factor above a given risk threshold - and the spread in this distribution that this mean conjunction metric describesdecreases significantly as the thrust level increases beyond $\sim 10 \mathrm{mN}$. Re-orbit maneuvers beneath this thrust level become more challenging in that larger $1 \sigma$ uncertainties make the number of conjunctions for a particular set of initial conditions more difficult to forecast.

\subsection{Distribution of Conjunctions in Hill Frame}

For each of the conjunction events detected across all thrust accelerations considered in this study, it is beneficial to consider the distribution of these conjunctions in the Hill frame, ${ }^{12}$ which is centered on the re-orbit system and has axes directed in the orbit radial, in-track, and cross-track directions (Schaub and Junkins, 2009). For all conjunctions at a distance threshold of $50 \mathrm{~km}$ detected across all 1152 trajectories at a thrust acceleration of $5 \mathrm{mN}$, Figure 10 illustrates the distribution in the local orbit plane by relative position magnitude in Figure 10(a) and relative velocity magnitude in Figure 10(b). As Figure 10 highlights, trends in the relative position and velocity distributions are not observed, i.e., the conjunction events detected are uniformly distributed (qualitatively) in the radial/intrack and radial/cross-track planes. Furthermore, these conjunctions occur at a broad range of relative speeds that are uncorrelated with the location of these conjunctions in the Hill frame. No correlations in these Hill frame distributions with thrust level, initial longitude, initial inclination, or conjuncting debris object class are observed.

Figure 10 has important implications for guidance strategies for continuous thrust re-orbit systems that are equipped to attenuate conjunction risk by adjusting the thrust profile such

\footnotetext{
${ }^{12}$ The Hill frame is also referred to as the local vertical, local horizontal (LVLH) frame in the relative motion literature.
} 


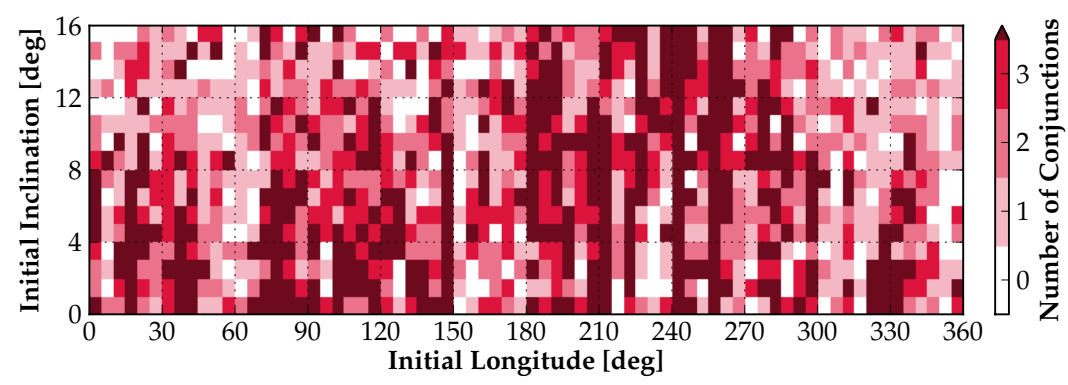

(a) Number of conjunctions at $50 \mathrm{~km}$ (thrust level: $1.0 \times 10^{-6} \mathrm{~m} / \mathrm{s}^{2}$ ).

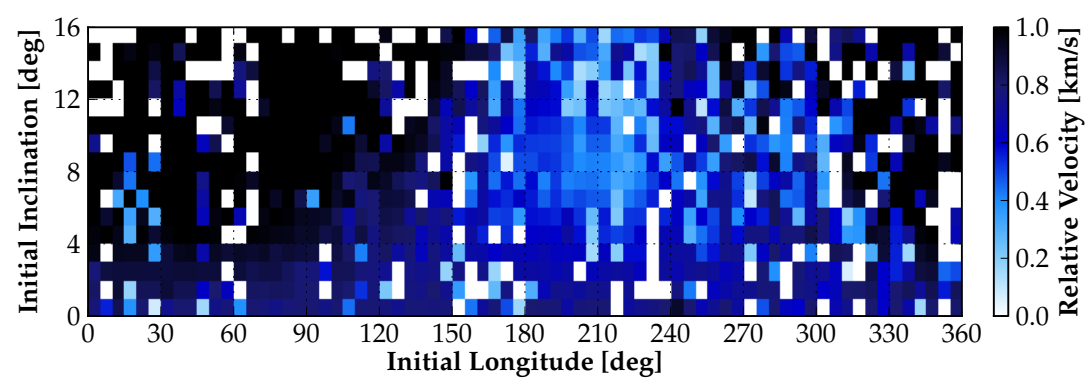

(b) Worst-case $50 \mathrm{~km}$ conjunction speeds (thrust level: $1.0 \times 10^{-6} \mathrm{~m} / \mathrm{s}^{2}$ ).

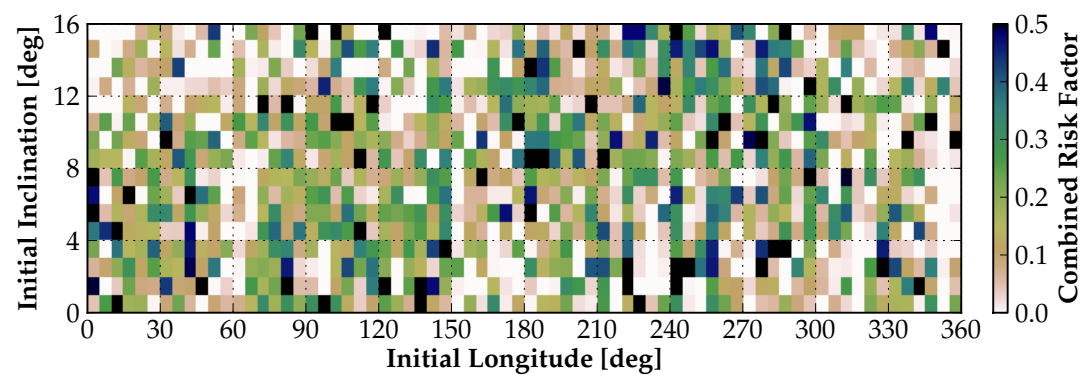

(c) Worst-case $50 \mathrm{~km}$ conjunction risk (thrust level: $1.0 \times 10^{-6} \mathrm{~m} / \mathrm{s}^{2}$ ).

Figure 8: Number of conjunctions at $50 \mathrm{~km}$ shown with worst-case relative speeds and combined risks for these trajectories, as a function of initial longitude and inclination. 


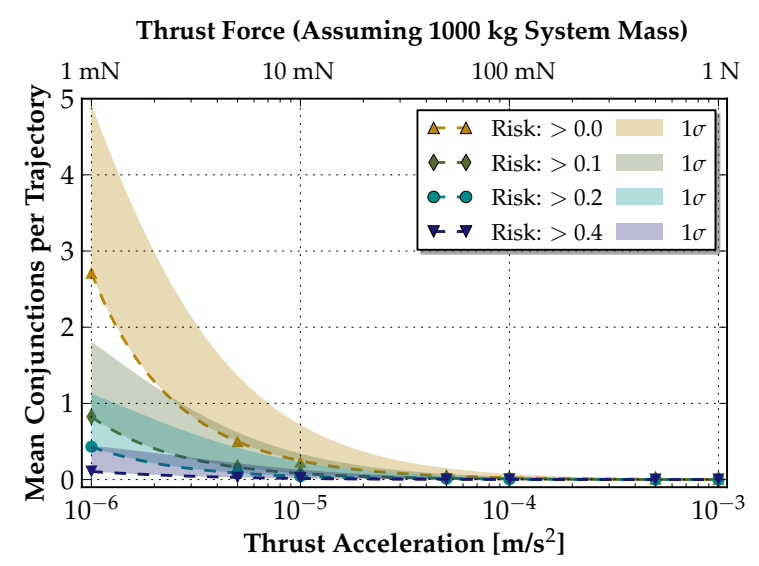

(a) Mean number of conjunctions with $1 \sigma$ swaths.

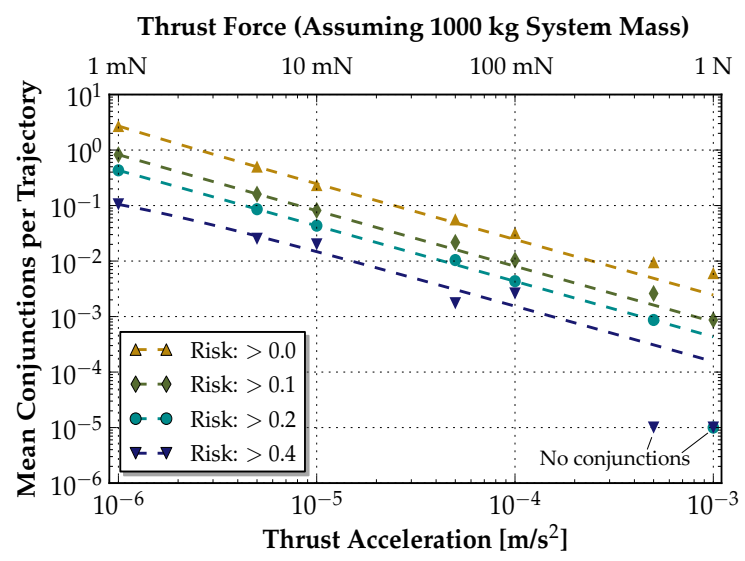

(b) Mean number of conjunctions (logarithmic $y$ axis).

Figure 9: Mean number of conjunctions per trajectory at $50 \mathrm{~km}$ as function of thrust level and risk, shown with hyperbolic best-fit lines on linear and logarithmic axes.

that a sufficiently evasive trajectory is achieved relative to a conjuncting debris object. Since no trends are qualitatively observed in the Hill frame position and velocity distributions, guidance strategies for avoidance must be flexible and robust, designed to handle any given relative position and velocity in the Hill frame. Note that the conjunctions illustrated in Figure 10 are the positions of conjuncting debris when these objects first come within the distance threshold of $50 \mathrm{~km}$, which is not necessarily the time of closest approach to the re-orbit system for each of these derelicts. Positions at the times of "first approach" are used to simulate the environmental sensing and detection performed by an autonomous re-orbit system, activities that would determine whether execution of the guidance strategy is even necessary.

\subsection{Effect of Delaying Start Time of Maneuver}

All of the re-orbit trajectories considered thus far in this study are initialized at the burn start epoch of 03/01/2014 (00:00:00 Zulu), but the "holes" present in the initial condition space in Figure 5 for each thrust level suggest that the number of conjunction events for a given trajectory can be reduced, or even eliminated, if the start time of the maneuver is delayed. To investigate the effect of delaying the start time for the 1152 trajectories propagated at each thrust level, Figure 11 provides the number of conjunction events at a distance threshold of $50 \mathrm{~km}$, for the $2 \mathrm{mN}$ thrust level, for start times in 6-hour increments over 03/01/2014. Studying Figure 11, it is evident that the number of conjunction events for the majority of trajectories can indeed be minimized or eliminated at particular times of day, which are dependent on the initial longitude - and therefore local time - of the re-orbit system.

Relative to an Earth-fixed observer, the collective motion of the GEO debris population is similar to a transverse wave with a period of one sidereal day (McKnight and Di Pentino, 2013), resulting from clustering in right ascension of the ascending node driven by luni-solar perturbations. McKnight and Di Pentino (2013) indicate that equatorial crossing windows 


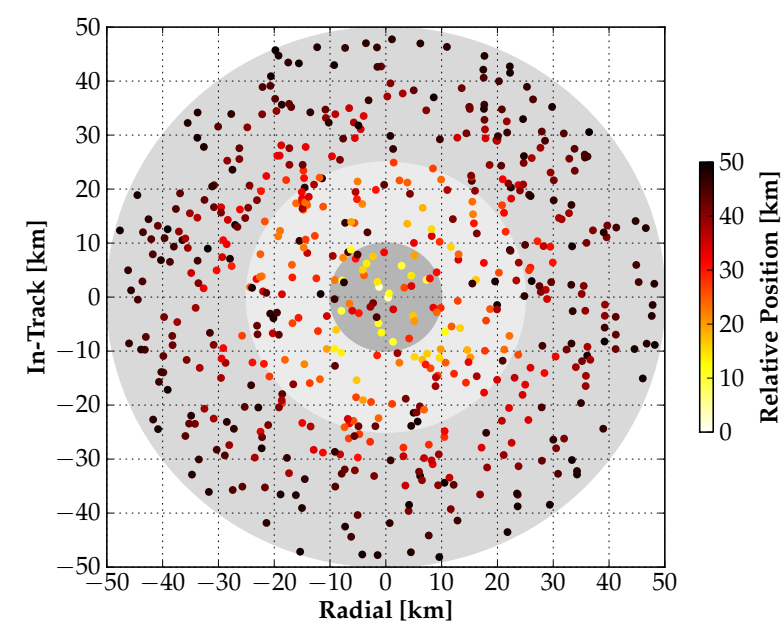

(a) Relative position distribution for $50 \mathrm{~km}$ conjunctions.

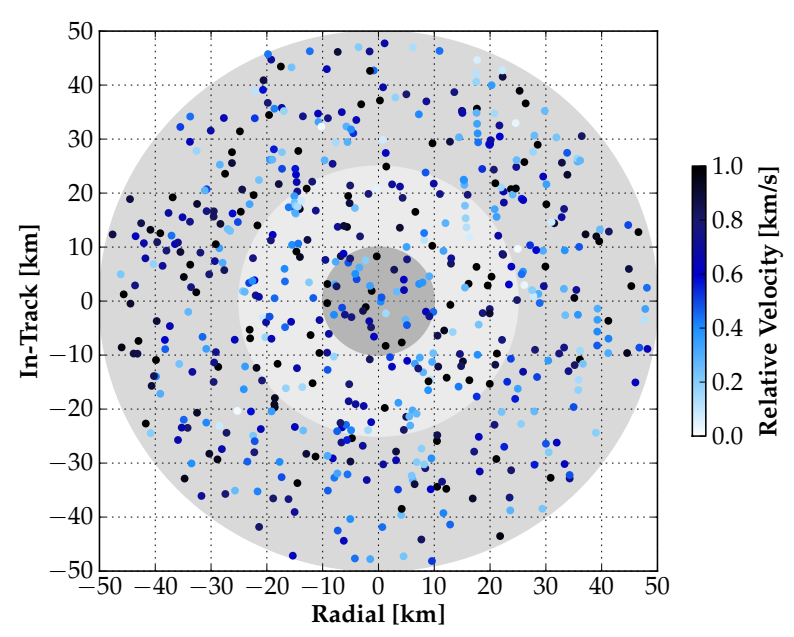

(b) Relative velocity distribution for $50 \mathrm{~km}$ conjunctions.

Figure 10: Distribution of conjunctions at $50 \mathrm{~km}$ in local Hill frame (thrust level: $5.0 \times 10^{-6} \mathrm{~m} / \mathrm{s}^{2}$ ).

of the GEO debris population are synchronized such that collision hazard to any longitude slot around the GEO ring is episodic and predictable, dependent on time of year in addition to time of day. Thus, delaying the start time for the re-orbit maneuver is analogous to shifting the latitudinal position of the synchronized debris population located at the initial longitude of the re-orbit system. Recalling that the re-orbit trajectories surveyed in this study are initialized at the ascending node in the equatorial plane, this suggests that trajectories beginning "in-sync" with local debris motion will experience more conjunctions than those beginning asynchronous to this transverse wave of debris, albeit with lower relative conjunction velocities.

Figure 12 shows the maximum relative velocities for the conjunction events at a distance threshold of $50 \mathrm{~km}$ illustrated in Figure 11. A prominent "wave-like" phenomenon that shifts westward nearly linearly across the longitude space as the start time is delayed over the course of one day is exhibited in Figure 12-a physical manifestation of the GEO debris synchronization effect on the conjunction challenges investigated in this study. As expected, the wave-like regions of minimum relative speed in Figure 12 correspond to the longitudes at which the local debris population is ascending through the equatorial plane from south to north at that time of day (McKnight and Di Pentino, 2013). Again, since all propagated trajectories begin at the ascending node in the equatorial plane, these longitudes of reduced relative velocity are those at which the re-orbit system is either partially or fully in-phase with local debris motion at the beginning of the transfer. Conversely, the darker regions of higher relative velocity in Figure 12 are those at which the re-orbit system begins out-of-phase with the local debris population - the re-orbit system rises in latitude from the ascending node as the local debris population descends in latitude from north to south through the equatorial plane, leading to increased relative speeds at conjunction.

To emphasize the importance of the start time of the re-orbit maneuver in reducing or eliminating the number of conjunction events for a given initial longitude and inclination, Figure 13 provides the number of conjunctions at a distance threshold of $50 \mathrm{~km}$ for two 


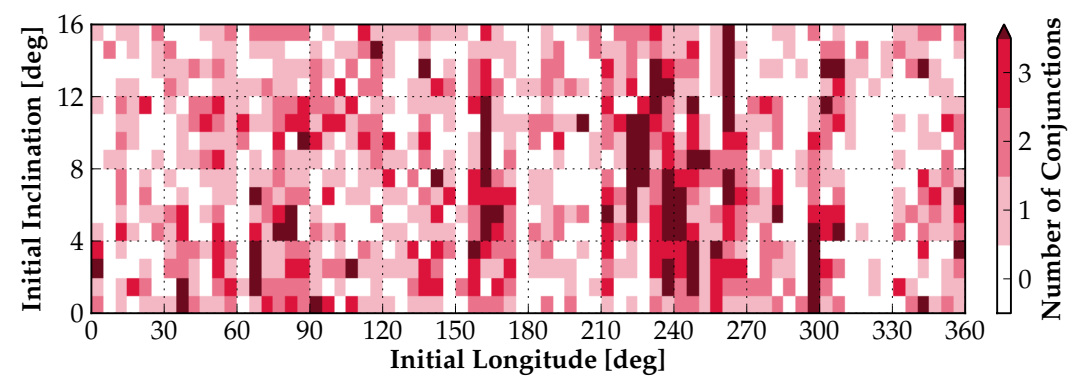

(a) Number of conjunctions at $50 \mathrm{~km}$ (re-orbit starts at 00:00:00 Zulu).

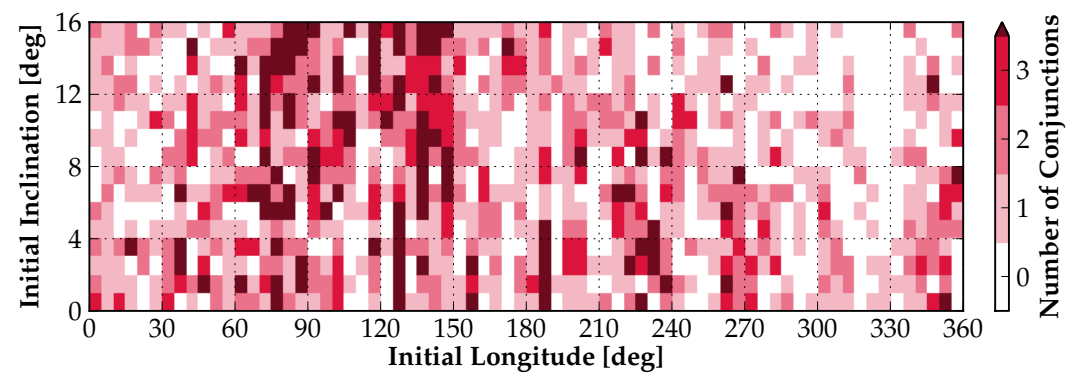

(b) Number of conjunctions at $50 \mathrm{~km}$ (re-orbit starts at 06:00:00 Zulu).

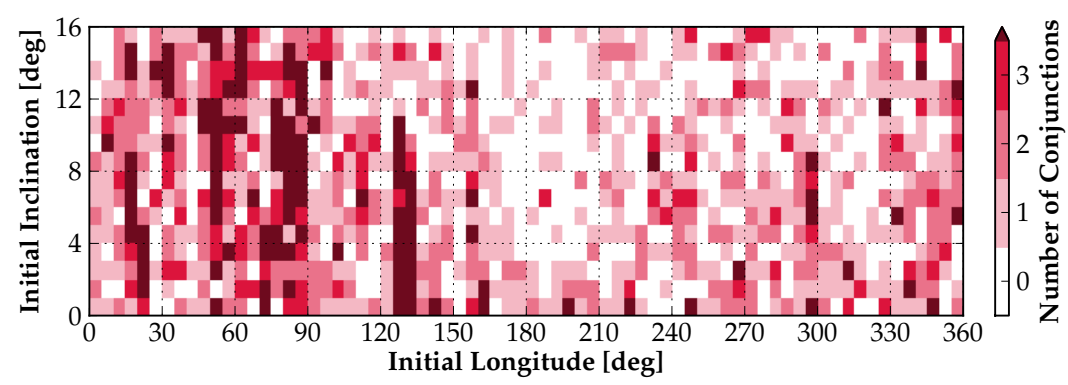

(c) Number of conjunctions at $50 \mathrm{~km}$ (re-orbit starts at 12:00:00 Zulu).

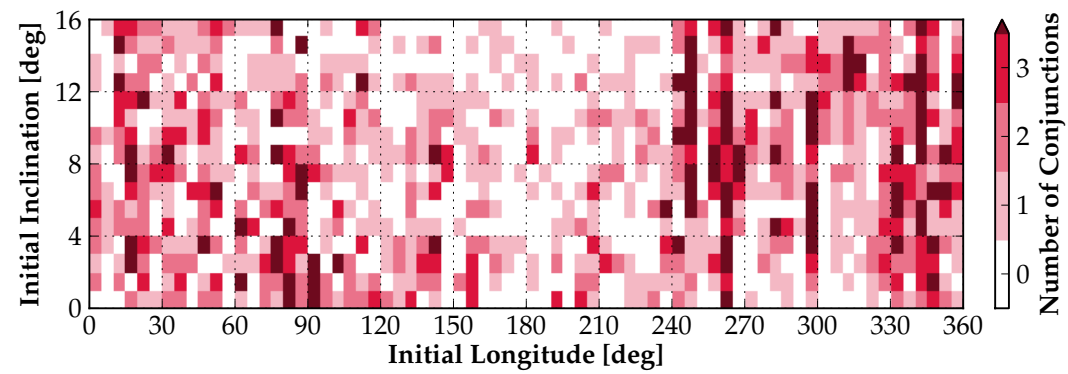

(d) Number of conjunctions at $50 \mathrm{~km}$ (re-orbit starts at 18:00:00 Zulu).

Figure 11: Effect of delaying start time for re-orbit maneuver (thrust level: $2.0 \times 10^{-6} \mathrm{~m} / \mathrm{s}^{2}$ ). 


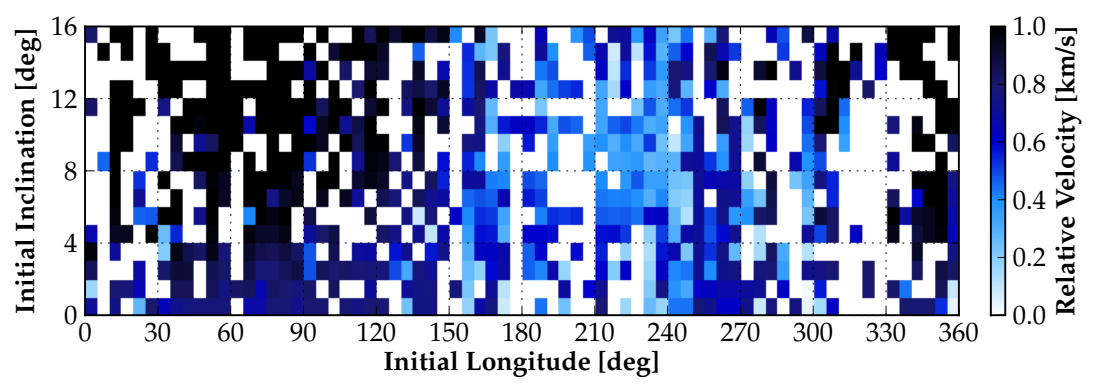

(a) Worst-case $50 \mathrm{~km}$ conjunction speeds (re-orbit starts at 00:00 Zulu).

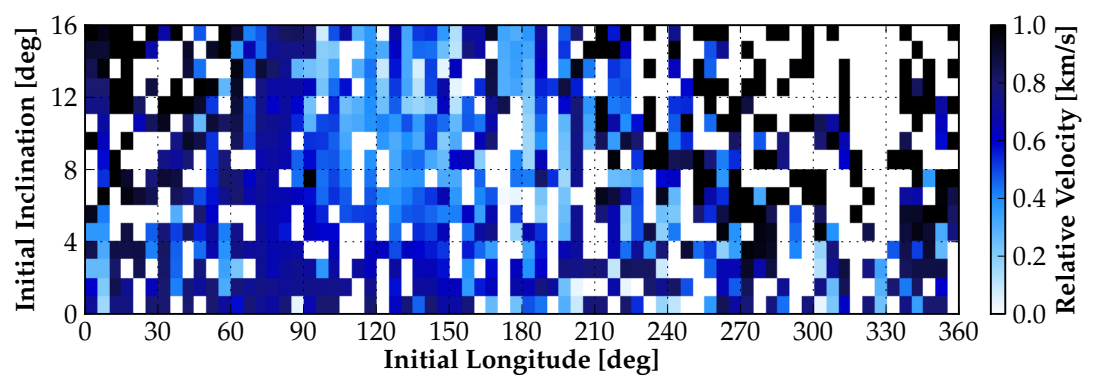

(b) Worst-case $50 \mathrm{~km}$ conjunction speeds (re-orbit starts at 06:00 Zulu).

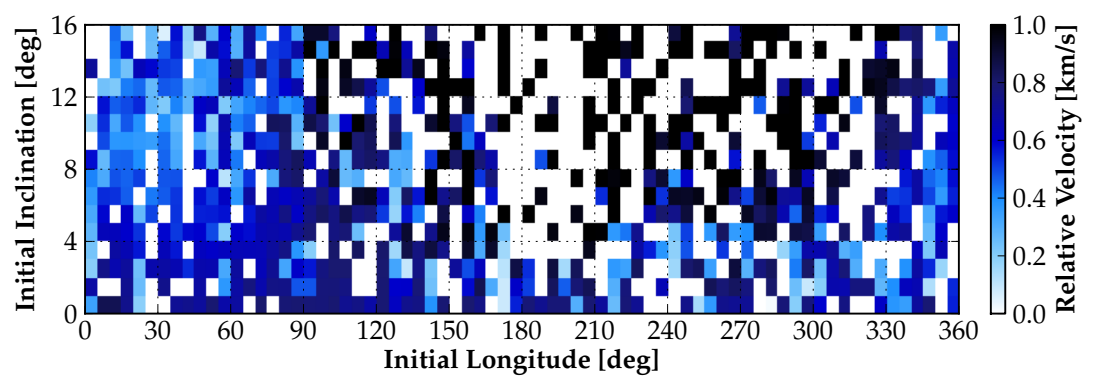

(c) Worst-case $50 \mathrm{~km}$ conjunction speeds (re-orbit starts at 12:00 Zulu).

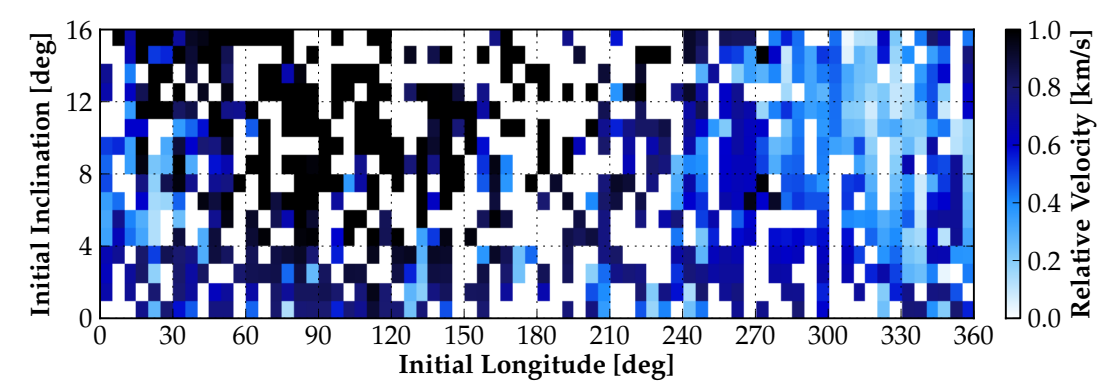

(d) Worst-case $50 \mathrm{~km}$ conjunction speeds (re-orbit starts at 18:00 Zulu).

Figure 12: Effect of delaying start time for re-orbit maneuver (thrust level: $2.0 \times 10^{-6} \mathrm{~m} / \mathrm{s}^{2}$ ). 


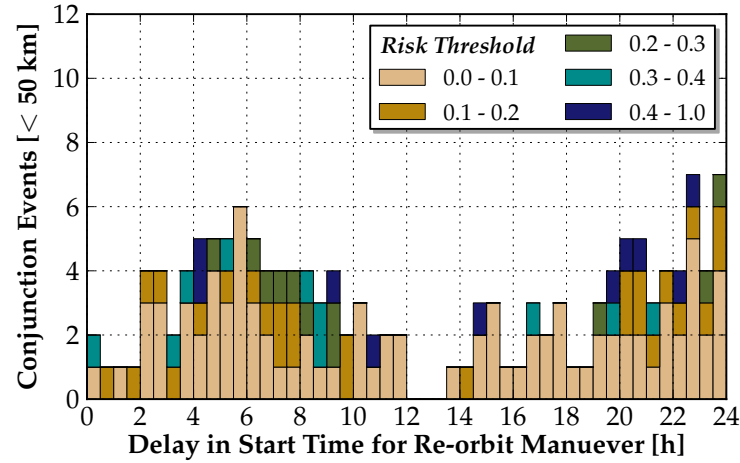

(a) Conjunctions at $50 \mathrm{~km}\left(\lambda_{0}=75^{\circ}, i_{0}=0^{\circ}\right)$.

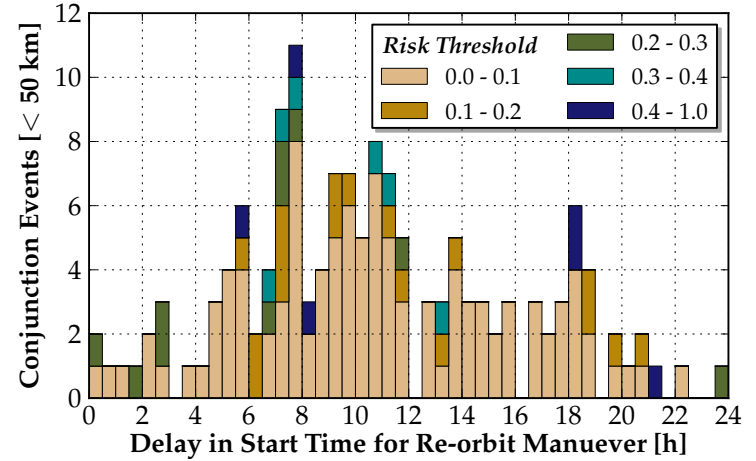

(b) Conjunctions at $50 \mathrm{~km}\left(\lambda_{0}=75^{\circ}, i_{0}=15^{\circ}\right)$.

Figure 13: Number of conjunctions for two trajectories as a function of maneuver delay time.

trajectories - $\left(\lambda_{0}, i_{0}\right)=\left(75^{\circ}, 0^{\circ}\right)$ in Figure $13(\mathrm{a})$ and $\left(\lambda_{0}, i_{0}\right)=\left(75^{\circ}, 15^{\circ}\right)$ in Figure $13(\mathrm{~b})$-as a function of maneuver delay in half-hour increments. The number of conjunction events at each delay time is further subdivided by combined risk level, to highlight that not only the number of conjunctions is subject to change under a delayed start time, but the risk factors for the anticipated conjunctions can change as well. For both cases in Figure 13, there exist optimal delay windows at which the number of conjunction events at a distance threshold of $50 \mathrm{~km}$ experienced over the entirety of the re-orbit maneuver are eliminated. Therefore, the timing for the re-orbit maneuver is critical, and should be chosen such that asynchronization with local debris motion at the beginning of the maneuver is leveraged to reduce - or even eliminate - the number of conjunction events experienced for a given thrust level over the duration of the maneuver.

\section{Conclusions}

The conjunction challenges of applying low-thrust engines for continuous thrust re-orbiting of GEO objects to super-synchronous disposal orbits are investigated in this study to inform design considerations for GEO debris mitigation and remediation technologies calling for lowthrust propulsion. The lower maneuverability of re-orbit systems equipped with lower-thrust engines increases the challenges of collision avoidance, such that quantifying the number of potential conjunction events experienced with the current GEO debris population during a typical re-orbit to $300 \mathrm{~km}$ above GEO is an imperative task. In particular, a two-dimensional sweep over initial longitude and inclination is performed, in which surveyed trajectories are propagated from the GEO ring under various in-track thrust levels until the $300 \mathrm{~km}$ increase in semi-major axis is achieved. Then, conjunction events with large-scale GEO debris objects from the publicly-available TLE population are detected within various conjunction distance thresholds in post-processing.

Results demonstrate that the number of conjunction events experienced for a given thrust level and distance threshold increases hyperbolically on average as the thrust level decreases, in correlation with re-orbit maneuver duration as a function of thrust level. The contribution of this paper, however, is not to reaffirm that higher thrust levels result in fewer conjunctions along a GEO re-orbit trajectory, but to highlight particular conjunction challenges that arise 
when thrust levels beneath approximately $10^{-5} \mathrm{~m} / \mathrm{s}^{2}(10 \mathrm{mN}$ for a $1000 \mathrm{~kg}$ system mass) are considered. Sensitivities to the initial longitude and initial inclination of the re-orbit system at a particular start time, thrust level, and distance threshold are not observed. Examination of the conjunction events in semi-major axis and longitude space, however, highlights regions of this phase space in which conjunction events are more likely, e.g., around the gravitational wells at $75^{\circ} \mathrm{E}$ and $105^{\circ} \mathrm{W}$ within $50 \mathrm{~km}$ of the GEO ring in semi-major axis. Advantageously, timing the re-orbit maneuver appropriately - such that the re-orbit system is out-of-phase at the start of the transfer with the wave-like behavior of Earth-relative debris motion at GEOserves to reduce, or possibly eliminate, the conjunctions anticipated for a given trajectory at a given thrust level. No conjunctions within a distance threshold of $25 \mathrm{~km}$ are detected across all surveyed trajectories at the $5 \times 10^{-4} \mathrm{~m} / \mathrm{s}^{2}$ and $10^{-3} \mathrm{~m} / \mathrm{s}^{2}$ levels, indicating that timing is less necessary for thrust levels above these cases. However, inasmuch as the debris population at GEO continues to increase in the absence of global adherence to mitigation guidelines - and since debris objects smaller than approximately one meter are not considered in this study - it is critical that GEO re-orbit systems be equipped with a robust guidance subsystem to adjust the vehicle's thrust profile in the event that timely evasion of conjuncting debris is warranted.

\section{Acknowledgments}

The authors would like to acknowledge the U.S. Department of Defense and the National Defense Science and Engineering Graduate Fellowship (NDSEG), the program through which funding for this research was obtained. The authors furthermore acknowledge Brandon Jones and the TurboProp software suite (Hill and Jones, 2009), from which the integration routines employed in this research were obtained. Finally, the authors would like to thank the anonymous reviewers who strengthened the content of this manuscript.

Anderson, P. V., Schaub, H., February 2014. Local debris congestion in the geosynchronous environment with population augmentation. Acta Astronautica 94 (2), 619-628.

Anderson, P. V., Schaub, H., 2016. Methodology for characterizing high-risk orbital debris in the geosynchronous orbit regime. Advances in Space Research 57 (2), 604-619.

Berry, M. M., Healy, L. M., July-September 2004. Implementation of gauss-jackson integration for orbit propagation. Journal of the Astronautical Sciences 52 (3), 331-357.

Bombardelli, C., Pelaez, J., 2011. Ion beam shepherd for contactless space debris removal. Journal of Guidance, Control and Dynamics 34 (3), 916-920.

Chrystal, P., McKnight, D., Meredith, P., 2011. Space debris: On collision course for insurers? Tech. rep., Swiss Reinsurance Company Ltd.

Flohrer, T., February 2014. Classification of Geosynchronous Objects: Issue 16. Tech. Rep. 1, European Space Operations Centre.

Hanada, T., Yasaka, T., Hata, H., Akahoshi, Y., September-October 2005. Using NASA Standard Breakup Model to Describe Low-Velocity Impacts on Spacecraft. Journal of Spacecraft and Rockets 42 (5). 
Hansen, B. W., Sorge, M. E., August 2013. Summarizing the general effects of breakup debris in geo. In: Proceedings of the 2013 AAS/AIAA Astrodynamics Specialist Conference. No. $13-844$.

Hill, K., Jones, B. A., May 2009. TurboProp Version 4.0. Colorado Center for Astrodynamics Research, University of Colorado at Boulder.

Hoots, F. R., Roehrich, R. L., December 1980. Spacetrack report no. 3: Models for propagation of norad element sets. Tech. rep., Office of Astrodynamics, Aerospace Defense Center.

Jehn, R., Agapov, V., Hernandez, C., 2005. The situation in the geostationary ring. Advances in Space Research 35, 1318-1327.

Jehn, R., Hernandez, C., 2001. International practices to protect the geostationary ring. Space Debris 1, 221-233.

Johnson, N., 1999. Protecting the geo environment: Policies and practices. Space Policy 15, $127-135$.

Johnson, N. L., Krisko, P. H., Liou, J.-C., Anz-Meador, P. D., 2001. Nasa's new breakup model of evolve 4.0. Advances in Space Research 28 (9), 1377-1384.

Klinkrad, H., 2006. Space Debris: Models and Risk Analysis. Praxis Publishing.

McKnight, D. S., July-August 1991. Determination of Breakup Initial Conditions. Journal of Spacecraft and Rockets 28 (4), 470-477.

McKnight, D. S., Di Pentino, F. R., 2013. New Insights on the Orbital Debris Collision Hazard at GEO. Acta Astronautica 85, 73-82.

Montenbruck, O., Gill, E., 2000. Satellite Orbits: Models, Methods, Applications. Springer.

NASA, September 2009. Process for limiting orbital debris. NASA-STD-8719.14 Change 4, National Aeronautics and Space Administration.

Prussing, J. E., Conway, B. A., 2013. Orbital Mechanics, 2nd Edition. Oxford University Press.

Raballand, F., Mignon, D., Gonnaud, J.-L., April 2005. Eurostar 2000 disposal on a graveyard orbit: Orbit control strategy and operational implementation. In: Proceedings of the 4th European Conference on Space Debris. ESA/ESOC.

Schaub, H., Jasper, L., August 2011. Circular orbit radius control using electrostatic actuation for 2-craft configurations. In: Proceedings of the 2011 AAS/AIAA Astrodynamics Specialist Conference.

Schaub, H., Junkins, J. L., 2009. Analytical Mechanics of Space Systems, 2nd Edition. American Institute of Aeronautics and Astronautics, Inc. 
Schaub, H., Moorer, D. F., 2012. Geosynchronous large debris reorbiter: Challenges and prospects. Journal of the Astronautical Sciences 59 (1-2), 165-180.

Vallado, D., 2007. Fundamentals of Astrodynamics and Applications, 3rd Edition. Microcosm Press.

Vallado, D. A., Crawford, P., Hujsak, R., Kelso, T. S., August 2006. Revisiting spacetrack report no. 3: Revision 2. In: Proceedings of the 2006 AIAA/AAS Astrodynamics Specialist Conference. 